\title{
Affections buccales classantes dans l'infection à VIH. Etude prospective dans le Service des Maladies infectieuses du CHRU de Clermont-Ferrand
}

\author{
Saleh-Jawiche $\mathbf{M}^{1}$, Devoize $\mathbf{L}^{2}$, Jacomet $C^{3}$, Antoine $\mathbf{L}^{2}$, Huard $C^{2}$, Sudrat $\mathbf{Y}^{2}$, Deschaumes $C^{2}$, \\ Baudet-Pommel $\mathbf{M}^{2}$ \\ ${ }^{1}$ Service d'Odontologie, CHRU, Limoges, France \\ ${ }^{2}$ Service d'Odontologie, Sous-section Médecine buccale Chirurgie buccale, CHRU Hôtel Dieu, \\ Clermont-Ferrand, France \\ ${ }^{3}$ Service des Maladies infectieuses, CHRU Gabriel Montpied, Clermont-Ferrand, France \\ clermontois@hotmail.com
}

L'infection par le VIH reste, 30 ans après sa première description, un problème de santé mondial. Malgré l'avènement des thérapies anti-rétrovirales hautement efficaces (HAART), cette infection conduit inéluctablement à une défaillance du système immunitaire avec apparition d'infections opportunistes et/ou de tumeurs, notamment dans la sphère bucco-pharyngée.

Différents facteurs interviennent de manière efficace dans l'inhibition du VIH au niveau buccal. L'immunité cellulaire, les anticorps anti-viraux ou les protéines salivaires non spécifiques (lactoferrine, lysozymes, PRP, SLPI...) font de la cavité buccale un rare site de transmission, contrairement à ce que l'on observe sur les autres muqueuses, en particulier génitales.

Mais lorsque l'infection virale est en échappement, les conséquences immunitaires sont majeures et multiples. On note une diminution des lymphocytes T CD4+ (LTCD4+), des macrophages et des cellules de Langherans, aboutissant ainsi à une susceptibilité accrue aux co-infections et aux lésions tumorales (Okunseri et al. 2003).

Notre étude avait pour objectif d'identifier les caractéristiques buccales des patients infectés par le VIH avec mise en évidence d'éventuels liens entre ces lésions et différents paramètres (âge, mode de contamination, variables immunitaires, addictions diverses, en particulier tabagique).

Notre étude a porté sur un total de 136 patients répartis selon les 3 stades de la maladie : 57 au stade A, 39 au stade B et 40 au stade C.

Prés de la moitié des patients présentaient une addiction tabagique importante. Au total, 50 patients présentaient une ou plusieurs lésions buccales dont $80 \%$ étaient constituées par des candidoses.

Nos résultats mettent en évidence des associations significatives connues entre développement d'une candidose bucco-pharyngée et augmentation de la charge virale avec diminution des LTCD4+ (Challacombe et al. 1997, Chattopadhayay et al. 2005, Sroussi et al. 2007). Néanmoins, les patients présentant une candidose, qu'ils soient sous traitement anti-rétroviral ou non, que leur charge virale soit élevée ou non et/ou que leur taux de LTCD4+ soit bas ou non, étaient en grande majorité des fumeurs. Les facteurs prédisposant à l'apparition de la candidose sont donc soit la déplétion immunitaire, soit l'addiction tabagique ou les deux à la fois.

Il ressort ainsi que la candidose reste la principale affection diagnostiquée chez les patients séropositifs pour le VIH. Son apparition reste fonction des facteurs classiques liés à l'immunité mais d'autres facteurs jouent un rôle dans l'apparition de cette lésion, notamment les habitudes addictives tabagiques. Tous ces résultats conduisent à considérer comme primordial et systématique l'arrêt du tabac dans la prise en charge thérapeutique des patients séropositifs. 